\title{
I. Abteilung.
}

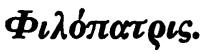

II.

(Vgl. Byz. Ztschr. V 1 ff.)

Was R. Crampe in dieser Zeitschrift Bd VI S. 144 ff. zur Empfehlung seiner Datierung des Pseudolucianischen $\Phi_{\iota} \lambda$ ó $\pi \alpha \tau \iota \iota s$ vorbringt, dürfte kaum geeignet sein, solche Leser, die sich durch eignes Studium mit dem Gegenstand vertraut gemacht haben, der Crampeschen Ansicht geneigter zu machen. Gleichwohl mag es nicht überflüssig sein, den ganzen Handel und die in ihm ausschlaggebenden Momente, die in dem Helldunkel, in dem sie bei Crampe flüchtig vorgewiesen werden, an Deutlichkeit nicht gewonnen haben, nochmals in scharfes Licht zu rücken. Die Wahrheit wird dann um so einleuchtender hervortreten.

Was Gutschmid (Kl. Schr. V 433 f.) bestimmte, den Philopatris in die Zeit des Kaisers Herakleios (610-641) zu setzen, war vornehmlich der Umstand, dafs sich unter dessen Regierung, den Anspielungen des Dialogs entsprechend, nicht nur Kämpfe mit „Persern“ und „Skythen" (dergleichen freilich Jahrhunderte lang ungefähr jeden oströmischen Kaiser beschäftigten), sondern daneben auch eine feindliche Invasion auf Kreta nachweisen liefs. Im Jahre 623 überfielen slavische Kriegerscharen Kreta und andere griechische Inseln.

Eine unbedingt sichere Datierung des Dialogs ergiebt sich aus diesen Momenten auf keinen Fall. Auch unter Konstantin II Pogonatos (668-685) trafen die gleichen Verhältnisse zusammen: schwere, endlich siegreiche Kämpfe mit den Orientalen, bedrohliche Regungen der „Skythen“, d. h. Slaven und Bulgaren, und im Jahre 674 ein Einfall der Araber in Kreta (s. Theophanes, Chron. I 354, 20 ed. de Boor; daraus Anastasius, hist. tripert. ibid. II 223, 32). ${ }^{1}$ )

1) Schon 653 ein arabischer Raubzug nach Kos, Kreta und Rhodus: Muralt, Chronogr. byzant. p. 709.

Byzant. Zeitschrift VI 3 u. 4. 
Es ist aber unmöglich, die Anspielungen des $\Phi_{\iota} \lambda \delta^{\prime} \pi \alpha \tau \varrho \iota s$ auf diese oder jene Affaire zu beziehen. Der Verfasser des Dialogs lälst eine seiner Figuren sich mit Behagen daran erinnern, wie kürzlich auf Kreta unzählige Jungfwen niedergemetzelt worden seien, und wie er selbst beliebig viele Jungfrauenhäupter von dort hätte mitbringen können (cap. 9). Dafs er dabei an die Häupter christlicher, griechischer Jungfrauen denke, die der ungläubige Feind abgehauen und er, der Grieche und Christ, habe mit sich nehmen können und mögen, wird sich durch Crampe niemand einreden lassen. Wie soll man es sich auch nur möglich denken, dafs die Besiegten (die Byzantiner) die Häupter der von den Siegern (den Slaven) gemordeten Jungfrauen hätten mitnehmen können? Es ist ganz unleugbar von einem Gemetzel die Rede, in dem die Byzantiner Sieger waren und unter der auf Kreta angesiedelten andersgläubigen Bevölkerung ein Blutbad anrichteten, wie jetzt wieder ihre Nachkommen unter den Türken. Von einem solchen Sieg der Byzantiner auf Kreta unter Herakleios oder unter Konstantin Pogonatos wissen wir nichts; ja bei den Feinden, denen sie damals dort gegenuberstehen mochten, fanden die Griechen Jungfrauen überhaupt nicht vor, die sie hätten milshandeln können: jene waren nur auf Raubzaigen nach Kreta gekommen, auf denen sie Weiber ohne Zweifel so wenig mit sich fuhrten, wie jene spanischen Araber, die im Jahre 823/4 unter Abu-hafs die Insel heimsuchten. ${ }^{1}$ )

Im $\Phi_{\iota \lambda} \delta \pi \alpha \tau \rho \iota s$ ist also von einem kürzlich ausgefochtenen grolsen

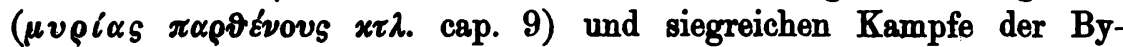
zantiner gegen eine auf Kreta fest angesiedelte moslimische Bevölkerung (von Fremden haben nur die Araber sich in jenen Zeiten dauernd auf Kreta angesiedelt) die Rede, wie er unter Herakleios nicht stattfand, noch stattfinden konnte, auch nicht unter Konstantiv Pogonatos, und tiberhaupt nicht vor dem Jahre 961, in dem Nikephoros Phokas nach heftigen Kämpfen die Insel wieder zum Reiche brachte.

Mit welchen Redewendungen sich Crampe der Anerkennung dieses für seine Datierung des. Dialogs verhängnisvollen Faktums entziehen zu können vermeint, möge man bei ihm S. 148 sich ansehen. Ich darf es mir erlassen, ihre Haltlosigkeit erst noch einzeln nachzuweisen.

Ebensowenig wie das hier Besprochene passen alle übrigen Hindeutungen des Dialogs auf Verhältnisse und Ereignisse seiner Zeit und Umgebung in die Regierungszeit des Herakleios. Kritias erzählt

1) Von diesen wird ausdrücklich erzæhlt, wie sie Weiber und Kinder bei ihrem Raubzuge daheim gelassen hatten; nach der (legendarischen) List des Abuhafs, die sie auf Kreta za bleiben nötigte, nahmen sie sich dort neue Weiber. S. Genesius p. 47 Lachm., Zonaras 15, 24 u. a. 
(cap. 19-22), wie er in Konstantinopel auf offener Strafse eine gewaltige Menge $(\pi \lambda \tilde{\eta} \vartheta 0 \varsigma$ s $\pi \dot{\alpha} \mu \pi 0 \lambda v)$ von Menschen, sich bedrohlich zuflüsternd, in unzufriedener Bewegung angetroffen habe; wie dann die Menge an den Weissagungen eines alten Männchens sich erfreut habe, das die Herankunft eines neuen Kaisers verkündigte, der namentlich den Armen Schulden und Steuern tilgen und erlassen, oder, wie ein anderer (dem ein Eremit den Namen des künftigen Herrschers aus Hieroglyphen zv $^{2}$

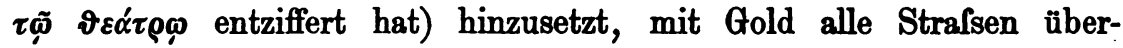
schwemmen werde. - Hier fand ich (Byz. Z. V 2) Spuren eines im Volke "tief aufgärenden Mifsvergnügens" mit dem bestehenden Regiment (vornehmlich, wie es in Despotien ohne politisches Leben zu sein pflegt, mit dessen fiskalischer Bedrickung), das in bedrohlicher Ankündigung des Sturzes der gegenwärtigen Herrschaft, zuletzt, wie das in cap. $23 \mathrm{ff}$. angedeutet wird, in einer Verschwörung gegen das Staatsoberhaupt sich Ausdruck giebt. Unter Herakleios bestand weder solche Unzufriedenheit, noch bereitete sich damals eine Verschwörung gegen den Kaiser vor. Crampe p. 149 findet das "selbstrerständlich“, meint aber, man habe doch an die Zeit des Herakleios zu denken, denn in dem Dialog sei nur von „Mifsstimmung einer kleinen, abseits stehenden Gruppe von Heiden" die Rede. So redet er angesichts der Schilderungen der cap. $19 \mathrm{ff}$, in denen uns $\varepsilon_{\nu} \tau \tilde{\eta} \lambda \varepsilon \omega \varphi \delta \rho \omega$ ein von Unglückspropheten aufgereiztes $\pi \lambda \tilde{\eta} \vartheta 0_{S} \pi \dot{\alpha} \mu \pi 0 \lambda v$ von Unzufriedenen gezeigt wird, das vom Heidentum auch nicht den Schatten einer verlorenen Spur zeigt. Was soll man dazu sagen? -

Nach den Meldungen von grofsen Siegen des Kaisers im Osten hofft Triephon, dafs die nächste Generation sehen werde $B \alpha \beta v \lambda \tilde{\nu} \alpha \alpha 3 \lambda$ -

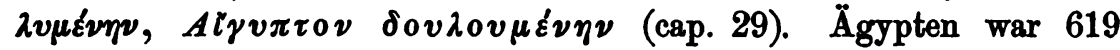
von den Persern erobert; 629 kam es wieder in römische Gewalt; schrieb der Verfasser des $\Phi$ เ $\lambda \delta \pi \alpha \tau \rho \iota s$ (wie Crampe annimmt) in der

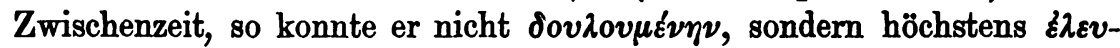

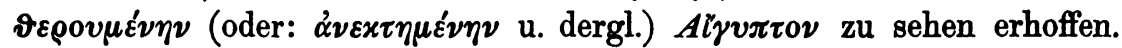
Dals in Ägypten, wie Crampe p. 149 hervorhebt, die Jakobiten in der Mehrzahl waren, konnte zwischen 619 und 629 doch wahrlich niemanden veranlassen, den gehofften Wiederanfall der noch keineswegs aufgegebenen, der Hauptstadt für ihre Verproviantierung unentbehrlichen, durchaus von Christen bewohnten römischen Provinz eine „Knechtung“ derselben durch ihre nächsten Angehörigen, die Römer, zu nennen. Mit welchem Namen hätte dann vollends der damals noch bestehende Zustand einer Unterwerfung unter Persien benannt werden

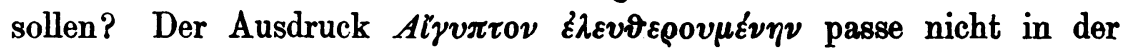

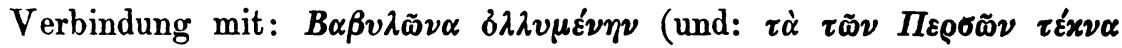




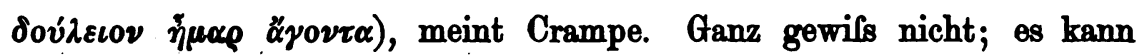

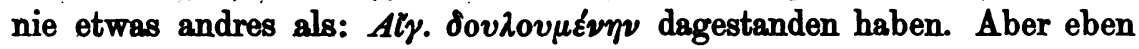
daraus folgt $\mathrm{ja}$, und habe ich geschlossen, dafs die Worte nicht unter Herakleios geschrieben sein können, zu dessen Zeit (vor 629) man

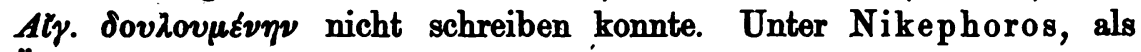
Ägypten längst den Römern ganz entfrumdet war, heifst es mit vollem

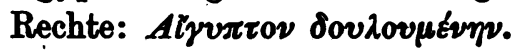

Sollte die Schrift unter Herakleios verfafst sein, so mülste das vor 629 geschehen sein, zu einer Zeit, als Ägypten noch nicht wieder dem Reiche angehörte. Damals konnte aber niemand, um der Hoffnung des vollständigen Sieges über den östlichen Feind den höchsten und abschliefsenden Ausdruck zu geben, darauf verfallen, zu sagen: $\pi \varepsilon \sigma \varepsilon i \tau \alpha \iota$

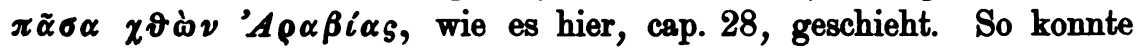
man erst reden, seitdem die Araber ein weites Gebiet sich erobert hatten und die gefährlichsten Feinde der Byzantiner im Osten geworden waren. 629 war hiervon noch nichts zu spüren. Und wiederum in den späteren Jahren der Regierung des Herakleios (die aber ubberhaupt nicht in Betracht kommen) konnte man wohl von dem gewaltigen Vordringen der Araber sagen, aber nur ein Verrückter hätte damals, als ihr ungestümer Siegeslauf noch nirgends eine Hemmung fand, von einer nahe bevorstehenden Zertrtimmerung des "ganzen Gebietes Arabiens" träumen können. Auf die Zeit des Herakleios pafst hier nichts; desto vollständiger parst alles auf die Zeit des Nikephoros Phokas, in der man, dem schon stark geschwächten und zersplitterten Reiche der

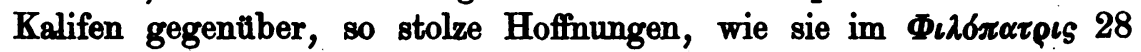
sich laut machen, nicht nur sehr wohl hegen konnte, sondern, wie Byz. Z. V 5 nachgewiesen, thatsächlich hegte und äu(serte. $\left.{ }^{1}\right)$ Es giebt in der byzantinischen Geschichte vor den Zeiten der makedonischen Dynastie, und insbesondere vor der Herrschaft des Nikephoros, überhaupt keinen Zeitpunkt, in dem solche Hoffnungen hätten geäufsert werden können.

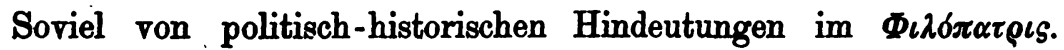
Andere, solche, die sich aus den Verhältnissen der Zeit des Herakleios verstehen liefsen, fehlen gänzlich.

Es bliebe also jedenfalls unmöglich, in diese Zeit die Abfassung des Dialogs zu verlegen, auch wenn es sonst Gründe gäbe, welche die Verlegung der Schrift in möglichst frühe Zeit, in eine Zeit, in der es

1) Mat den dort angeführten Worten des Abulfaragius vgl. noch, was, bei Gelegenheit der Kriegsthaten des Nikephoros, Georgios Hamartolos prahlt (p. 861

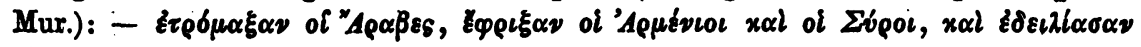

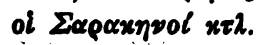


noch lebendiges Heidentum in Konstantinopel gab, rätlich machten. Crampe, dem die ganze Schrift als „eine Streitschrift der Kirche gegen das hellenische Heidentum" erscheint, sieht so gut im ersten Teil (cap. 1-18) wie im zweiten Teil (cap. 19 ff.) eine ernsthafte Bekämpfung heidnischen Glaubens und eine auf Restabilierung der alten Religion gerichtete Verschwörung. Hierüber brauche ich, nach den Ausführungen des ersten Artikels (Byz. Z. V $10 \mathrm{ff}$.), die Crampe völlig unangefochten gelassen hat, nicht viele Worte zu machen.

Der erste Teil mit seinem scherzhaften Geplänkel eines angeblichen Heiden mit einem kürzlich durch den Apostel Paulus dem Christenglauben Gewonnenen und Getauften (cap. 12) ist nichts als eine litterarische Posse, ohne ernste und praktische Tendenz und selbst ohne die Absicht, eine ernste Tendenz aufmerksamen Lesern vorzutäuschen. Crampe, der dies, seltsam genug, verkennen konnte, findet hier eine vollkommen ernst und bieder gemeinte „religiöse Polemik“ und wird allein durch dieses Mifsverständnis bewogen, nun auch für den zweiten Teil sich das Motiv einer religiösen Polemik auszudenken, das sich . dort vollends gar nirgends blicken lassen will. Die possenhafte Heidenbekehrung des ersten Teils verlegt der Verfasser in die Zeit der ersten Christen: vom Apostel Paulus ist Triephon, der hier den Kritias bekehrt, getauft. Das kann, nach meinem Hinweis (Byz. Z. V 12), Crampe p. 147 nicht leugnen; über die für seine Datierung unangenehmen Konsequenzen dieser Wahrnehmung sucht er sich hinwegzuhelfen mit dem Rätselwort: „Kritias wird sicher seinen Freund hinreichend gekannt haben, um sich ihm anvertrauen zu können, sodals (??) man in der Heraufbeschwörung des Apostels Paulus als Bekehrers des Triephon keine Maskerade zu sehen braucht; es ist einfach eine Pose des Schriftstellers, der seine Belesenheit zeigen will." Dunkel ist der Rede Sinn; was sich aber nicht verdunkeln läfst, ist der Sinn und Grund der Verlegung dieser Heidenbekehrung in eine ferne Vergangenheit: hierdurch bezeugt der Verfasser auf das deutlichste, dafs er an eine Polemik gegen ein noch in seiner Zeit aufrechtstehendes Heidentum nicht denkt, gar nicht denken kann, weil es so etwas in seiner Zeit längst nicht mehr gab. Das einzige Argument für die Verlegung des Dialogs in die Zeit des Herakleios: dafs es damals in Konstantinopel noch Heiden gegeben habe, die man habe bekämpfen können (was nicht einmal richtig ist: s. Byz. Z. V 10 f.), fällt also gänzlich über den Haufen: der Verfasser sagt es selbst, dafs er in seiner Zeit ein Heidentum weder kenne, noch zu bekämpfen brauche.

Der zweite Teil,' cap. $19 \mathrm{ff}$., läfst von Heidentum der Unzufriedenen und Verschworenen nicht die allerleiseste Spur erkennen: und 
doch soll nach Crampe die ganze, hier von dem Verfasser des Dialogs denunzierte Verschwörung ihren Hauptzweck in einer Wiederherstellung des Heidentums haben. Heiden sollen sowohl (nach Crampe 146) „die auf der Strafse miteinander flüsternden untergeordneten Mitglieder des Konventikels" sein (es ist, wie schon gesagt, ein $\pi \lambda \tilde{y} \vartheta 0 s \pi \alpha u \mu \pi 0 \lambda v$, das da miteinander munkelt; woher Crampe die Nachricht hat, dafs diese gewaltige Menge aus Mitgliedern eines "Konventikels" bestehe, und zwar aus "untergeordneten“, bleibt sein Geheimnis), als ganz besonders die cap. $23 \mathrm{ff}$. vorgeführten Astrologen, die nach Crampe (146) einen „durch religiöse Handlungen geweihten Bund" miteinander bilden. Von

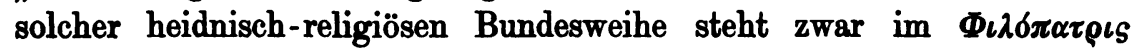
keine Sylbe zu lesen; aber, so beruhigt uns Crampe (146), es „wäre dort sicher das religiöse Element stärker hervorgetreten", wenn nicht u. s.w. - Die Schrift, lesen wir bei Crampe 146, verrate „durch die Bitterkeit ihrer Angriffe einen praktischen Zweck"; nachher (S. 149) belehrt er uns: „dafs das Heidentum des Konventikels nicht deutlicher hervortritt", sei ein Mangel, "der in den praktischen Tendenzen des Autors seine Erklärung finde." Wer sich darauf einen Vers machen kann, den möchte ich sehen. Die „praktische Tendenz" einer Denunxierung dieses angeblichen Heidenkonventikels soll in der "Bitterkeit ihrer Angriffe" dahin geführt haben, von dem Heidentum eben dieses "Konventikels" kein Wort zu sagen?

Denn so steht es ja: Heidentum tritt in den Worten und Handlungen der Unzufriedenen und der Verschworenen nicht etwa nur "weniger deutlich" hervor, wie sich Crampe euphemistisch ausdrückt, es ist darin gar nicht vorhanden ${ }^{1}$ ), wird auch nicht mit der diskretesten

1) Dafs die Unzufriedenen auf der Strafse und die Unglückspropheten, durch die sie aufgewiegelt werden, keine heidnischen Tendenzen haben konnen, hatte ich u. a. daraus entnommen (Byz. Z. V 10,1), dafs der Name des von ihnen gewünschten neuen Kaisers von einem christlichen Anschoreten aus Hiero-

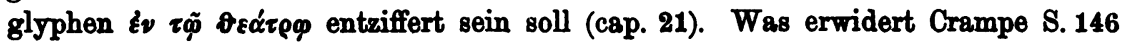
darauf? Ja, auch den Tod des Kaisers Maurikios habe, nach Theophyl. Simocatta VII 12 extr., ein Mönch auf offener Strafse geweissagt! Immer wieder diese Manier, wo es gilt, ein Argument des Gegners direkt zu bekampfen, von etwas ganz anderem zu reden. Ioh habe doch nicht an der Prophezeinng jenes Anachoreten als solcher Anstols genommen - nichts war ja gew 8 hnlicher in jenen Zeiten -, sondern daran, dafs ein christlicher Eremit eben den kommenden Kaiser verkündigt haben sollte, den sich eine Verschworung von Heiden wünschte. Dafür ist doch wahrhaftig der von Theophylakt erwăhnte Fall kein Beispiel. Dafs ein christlicher Eremit sich einen heidnisch gesinnten, den Heiden günstigen Kaiser nicht wünschen konnte, ist wohl einleuchtend, und dafs seine Weissagung nur der Ausdruok seiner Wünsche sein konnte, wird ja wohl auch nur bezweifeln können, wer sich etwa einen kindlichen Glauben an die Ob- 
Anspielung nur von ferne angedeutet. Wenn Crampe, der zu einem Nachweis thatsächlich vorhandener Spuren von Heidentum in jenen Scenen gar nicht einmal den schwächsten Ansatz macht, dennoch sich ïberzeugt hält, dafs er "das Heidentum des Astrologenvereins" auf das sorgfältigste nachgewiesen habe (S. 145), so begreift man das nur, wenn man bemerkt, wie er Beweis und Behauptung in einer sonderbaren Weise miteinander verwechselt. Behauptet hat er freilich in vielfach wechselnden Wendungen das Vorhandensein des nirgends wahrnehmbaren Heidentums der Verschworenen oft genug. Wollte man seinem Verfahren einen wissenschaftlichen Anstrich geben, so könnte man sagen, er führe die unbeweisbare Annahme des Heidentums jener Leute in seine Darstellung ein, wie der Dialektiker unter Umständen in -seine Beweiskette als obersten Satz eine $\vartheta \varepsilon \sigma \iota s ~ \alpha v \alpha \pi \delta \delta \varepsilon \iota x \tau 0$ s. Eine

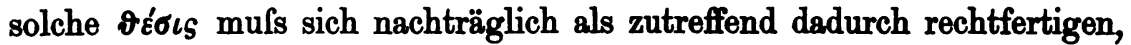
dafs nur bei ihrer Einführung die vorliegenden Erscheinungen ihre zu-

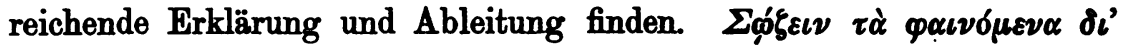
vं rade umgekehrt: wollte man bei den Verschworenen und Unzufriedenen heidnischen Glauben und heidnische Gesinnung voraussetzen, so würde alles, was von ihrem Gebaren und Reden gesagt wird, unverständlich und absurd. Der Leser des Dialogs könnte sich davon leicht überzeugen. Eine beliebige $\vartheta \varepsilon ́ \sigma \iota s, ~ e i n ~ s e l b s t e r d a c h t e r ~ H e i s c h e s a t z$ war überhaupt hier gar nicht einzusetzen, wo in der Kette von Grund und Folge nirgends eine Lücke ist, in die eine solche $\vartheta$ t́øı eintreten könnte, vielmehr die rein politischen Motive der Unzufriedenheit und Verschwörung alles vollkommen zureichend erklären.

Die Einheit des Themas der beiden Teile des Dialogs, die Crampe in einer beiden gemeinsamen "religiösen Polemil" gefunden zu haben meinte, besteht also nicht. Wie der Verfasser sogar die Einheit der Zeit spielend aufhebt, seinen Dialog bald in den Anfängen der Christenheit, bald in seiner eigenen Gegenwart, im zehnten christlichen Jahrhundert, sich bewegen läfst, so hat er in den beiden Teilen seiner Schrift von zwei ganz verschiedenen Dingen plaudern wollen, die durch keine andre Einheit als die des Verfassers und seiner bald mit diesem, bald mit jenem Spielzeug herumgaukelnden Eitelkeit zusammengehalten werden. Eine kritische Betrachtung kann diese Leichtfertigkeit der Komposition nur konstatieren; die mangelnde Einheit des Themas

jektivität solcher Gesichte und Seher bewahrt hat. Und da soll der christliche Anachoret eine Prophezeiung gegeben haben, die heidnische Verschworer sich, wie Crampe versichert, „zu nutze machten, weil sie ihnen so pafste"! Heiden konnte sie gar nicht "passen". 
durch beliebige Fiktionen zum Schein herzustellen, das ist nicht Sache der Kritik - eher ihres Gegenteils.

Wie endlich alles dahin führt, den Dialog, der unter Herakleios unmöglich geschrieben sein kann, mit Niebuhr in der Zeit der grofsen Siege des Nikephoros Phokas geschrieben zu denken, ist in meinem ersten Artikel auseinandergesetzt. Crampe hat nicht in einem einzigen Punkte den dort geführten Nachweis auch nur zu bemängeln versucht. Und völlige Beistimmung hat dieser Nachweis, wie ich mit Genugthuung wahrnehme, gefunden in Krumbachers Geschichte der byzantinischen Litteratur, S. $459 \mathrm{ff}$. der zweiten Auflage. Dabei wird es denn wohl sein Bewenden haben.

Heidelberg, 19. März 1897.

Erwin Rohde. 